\title{
Evaluación del Peróxido de Hidrógeno en la Oxidación de Fenol con Hierro Soportado Sobre Tela de Carbón Activado
}

\author{
Juan C. Moreno ${ }^{(1)}$, Víctor M. Sarria ${ }^{(1)}$, Ángel D. Polo ${ }^{(1)}$ y Liliana Giraldo ${ }^{(2)}$ \\ (1) Facultad de Ciencias, Departamento de Química, Universidad de los Andes, \\ Carrera 1 No. 18 A10, Grupo de Investigación en Sólidos Porosos y Calorimetría. \\ (e-mail: jumoreno@uniandes.edu.co) \\ (2) Facultad de Ciencias, Departamento Química, Universidad Nacional-sede Bogotá, \\ Carrera 30 Calle 45, Ciudad Universitaria, Bogotá-Colombia
}

\section{Resumen}

Se estudia el empleo de una tela de carbón activado comercial, un carbón activado americano y un carbón activado químicamente con $\mathrm{KOH}$, para obtener un material como soporte catalítico mediante impregnación de hierro en solución, para la degradación catalítica de fenol con peróxido de hidrógeno (degradación Fenton). La oxidación se realizó a $42^{\circ} \mathrm{C}$, pH entre 2.0-2.5, a presión atmosférica, una concentración inicial de fenol de $0.01064 \mathrm{M}$ y $0.89 \mathrm{M}$ de $\mathrm{H}_{2} \mathrm{O}_{2}$. Se logra una relación de impregnación de 1,39g Fe/gCA sobre la tela de carbón activado. Durante la oxidación del fenol, se siguió el consumo de $\mathrm{H}_{2} \mathrm{O}_{2}$, como una medición indirecta de la cinética alcanzándose una concentración mínima de $0.002 \mathrm{M}$ de $\mathrm{H}_{2} \mathrm{O}_{2}$ por la tela y por el carbón activado americano. El trabajo muestra que la tela de carbón activado como soporte catalítico en la descomposición de fenol es aceptable y competitiva con otros materiales porosos convencionales.

Palabras claves: tela de carbón activado, soporte catalítico, oxidación de fenol, reacción Fenton

\section{Evaluation of the Hydrogen Peroxide in the Oxidation of Phenol with Iron Supported on Activated Carbon Cloth}

\begin{abstract}
This study presents the generation and evaluation of three different materials containing activated carbon and iron for the heterogeneous Fenton degradation of phenol. Three different materials: (i) commercial cloth containing activated carbon, and (ii) pellet activated carbon and (iii) a chemically activated carbon with $\mathrm{KOH}$, were explored to support iron by means of impregnation. These three materials were used for the catalytic degradation of phenol with $\mathrm{H}_{2} \mathrm{O}_{2}$. The oxidation was performed at $42^{\circ} \mathrm{C}, \mathrm{pH}$ between 2,0-2,5, atmospheric pressure, an initial phenol concentration of $0,01064 \mathrm{M}$ and $\mathrm{H}_{2} \mathrm{O}_{2}$ 0.89M. A relation of impregnation of $1,39 \mathrm{gFe} / \mathrm{gAC}$ is obtained on the activated carbon cloth. During the oxidation of phenol, the disappearance of the $\mathrm{H}_{2} \mathrm{O}_{2}$ was followed as an indirect indication of the kinetics, reaching a minimal concentration of $0.002 \mathrm{M}$ of $\mathrm{H}_{2} \mathrm{O}_{2}$ by the cloth and the pellet activated carbon. The study shows that the use of cloth activated carbon as catalytic support for the decomposition of phenol is competitive with other conventional porous materials.
\end{abstract}

Keywords: cloth activated carbon, catalytic support, phenol oxidation, Fenton reaction 


\section{INTRODUCCIÓN}

La oxidación catalítica de compuestos orgánicos biorecalcitrantes es una técnica viable empleada para la descontaminación de aguas residuales. Una de las técnicas más utilizadas es el sistema Fenton. Este sistema consiste en una mezcla de hierro y agua oxigenada que, en fase homogénea, reacciona formando radicales hidroxilo $\mathrm{HO}$. Este radical libre tiene un fuerte poder de oxidación y se forma por la descomposición catalítica del peroxido de hidrogeno en medio ácido (Esplugas, 2002; Pignatello, 2006).

En la última década se han empleado diversos materiales porosos para soportar el hierro y de esta manera favorecer la formación de radicales hidroxilos y evitar el sacrificio del catalizador tal como se menciona en la literatura (Lucking, 1998; Wang, 2005; Guélou, 2003; Quintanilla, 2005; Das, 2004).

En este estudio se evalúa el uso de una tela de carbón activado comercial impregnada con hierro como posible soporte catalítico, aplicado en la degradación de fenol asistida con peroxido de hidrogeno. Igualmente se compara su rendimiento con otros carbones activados utilizados en el mismo proceso y posteriormente se determina la factibilidad del empleo de la misma como soporte catalítico en procesos de oxidación.

\section{METODOLOGIA}

El material precursor utilizado en el desarrollo del material es el siguiente:

\section{-Carbón activado con $\mathrm{KOH}$ (mineral Manto} AGR-Cesar).

-Carbón activado peletizado (comercialmineral).

-Tela activada comercial Zorflex A (Inglesa).

1. La muestra de carbón activado granular (Manto AGR) de origen mineral (bituminoso) fue activado químicamente con $\mathrm{KOH}$ empleando un tiempo de impregnación de 15 horas a temperatura controlada. Se utilizó una relación en peso de carbón 1:2 con respecto a la cantidad de $\mathrm{KOH}$, luego se realizó el secado de este a una temperatura de $120^{\circ} \mathrm{C}$ hasta lograr un peso constante $y$ posteriormente se realizó el proceso de carbonización de la muestra impregnada a una temperatura de $900^{\circ} \mathrm{C}$ por un tiempo de 1 hora y un flujo de $200 \mathrm{~mL} / \mathrm{min}$ de $\mathrm{N}_{2}$; empleando un reactor de cuarzo a una velocidad de calentamiento de $15^{\circ} \mathrm{C} / \mathrm{min}$.

2. La muestra de carbón activado peletizado es un carbón comercial de origen mineral obtenido desde un carbón bituminoso.

3. La tela de carbón activado Zorflex tipo A es una tela comercializada por la industria inglesa Zorflex. Esta tela es utilizada en la indumentaria del ejército ingles para la protección contra compuestos tóxicos.

La caracterización de los soportes catalíticos se realiza mediante la obtención de las isotermas de adsorción de $\mathrm{N}_{2}$ a $77 \mathrm{~K}$ de cada soporte catalítico. Para ello se necesito como tratamiento previo desgasificar cada uno de estos hasta una temperatura de $250^{\circ} \mathrm{C}$ para la tela activada y $300^{\circ} \mathrm{C}$ para los carbones activados, a través del uso del Sortómetro Autosorb-3B.

El área superficial se deduce de las isotermas de adsorción de $\mathrm{N}_{2}$ a $77 \mathrm{~K}$, empleando el método BET (Brunauer, Emmett y Teller) en un rango comprendido de presiones relativas entre 0.05 y 0.35 . Para la determinación del volumen y la distribución de poro, la técnica empleada fue el método DR (Dubinin Radushkevich), (Levenspiel, 1996; Rodríguez F. 1996).

\section{Tratamiento previo del material}

Se consideró como tratamiento previo a la impregnación de hierro, realizar una desmineralización y posteriormente una sulfonación de los carbones activados de origen mineral para favorecer aún más la impregnación de hierro en la matriz carbonosa (Lucking, 1998; Quintanilla, 2005). Los materiales precursores utilizados para realizar este procedimiento fueron los siguientes:

\section{-Carbón activado peletizado (comercial).}

\section{-Carbón activado con KOH (granular).}

La desmineralización o lixiviación de las dos muestras de carbón activado se llevó a cabo con $\mathrm{HCl} 5 \mathrm{~N}$ a $18^{\circ} \mathrm{C}$ durante 24 horas con el objetivo de remover los cationes metálicos de los carboxilatos en la estructura carbonosa. Se utilizó una relación de $5 \mathrm{~mL} \mathrm{HCl}(5 \mathrm{~N}) / 1 \mathrm{~g}$ 
carbón activado, la cantidad de carbón activado con $\mathrm{KOH}$ utilizada, fue de $1 \mathrm{~g}$ mientras que el comercial peletizado fue de $10 \mathrm{~g}$.

Una vez realizada la desmineralización con $\mathrm{HCl}$, se sulfonan las muestras para mejorar la capacidad receptora del hierro en la superficie carbonosa, como también dar mayor disponibilidad de interacción sobre los grupos funcionales en la trayectoria de la catálisis implicada. Para ello se empleó $\mathrm{H}_{2} \mathrm{SO}_{4}$ concentrado al $96 \%$ a $18^{\circ} \mathrm{C}$ durante 1 hora con una relación de $5 \mathrm{~mL} \mathrm{H}_{2} \mathrm{SO}_{4}(96 \%) / 1 \mathrm{~g}$ carbón activado, la cantidad de carbón activado con $\mathrm{KOH}$ utilizada fue de $1 \mathrm{~g}$, mientras que para el comercial peletizado fue de $10 \mathrm{~g}$ manteniéndose así la misma cantidad y el mismo tipo de muestra empleada en el proceso anterior. Posteriormente las muestras se lavaron con agua destilada manteniendo el $\mathrm{pH}$ ácido y finalmente se filtraron y se pesaron para proceder a disponer de ellas en el siguiente proceso de impregnación de hierro.

\section{Impregnación con hierro}

Las muestras previamente desmineralizadas y sulfonadas se impregnaron con hierro a través del contacto con una disolución al 20\% en peso de $\mathrm{FeSO}_{4} .7 \mathrm{H}_{2} \mathrm{O}$ durante 5 días a $18^{\circ} \mathrm{C}$. El pH fue ajustado entre 2.0 y 2.8 mediante una solución de $\mathrm{H}_{2} \mathrm{SO}_{4} 0.50 \mathrm{M}$ para así mantener el hierro en solución evitando la precipitación de este. La disolución fue realizada en frascos de ámbar para evitar la exposición a la luz en la solución y así evitar las posibles reacciones de foto-Fenton. De igual manera se procedió a impregnar con hierro la tela activada comercial inglesa (Zorflex A).

Se realizó una curva de calibración de $\mathrm{Fe}^{+2}$ mediante espectrometría UV-VIS, a una longitud de onda de $510 \mathrm{~nm}$ para determinar la concentración de hierro en solución mediante el análisis de la solución precursora, llevándola a dilución empleando una solución $2 * 10^{-3} \mathrm{M}$ de 1,10-fenantrolina (Fortune, et. Al 1938), una solución de clorhidrato de hidroxilamina al 10\%, una solución $0.1 \mathrm{M}$ de acetato de sodio y $\mathrm{H}_{2} \mathrm{SO}_{4}$ concentrado.

\section{Oxidación catalítica de fenol}

Se evaluó el efecto catalítico de los carbones activados empleados considerando la impregnación de hierro sobre la estructura carbonosa, en presencia de peroxido de hidrogeno. La tabla 1 presenta las cantidades utilizadas de cada uno de los carbones activados empleados. Los experimentos de degradación se realizan usando $50 \mathrm{ml}$ de una solución de fenol $0.01064 \mathrm{M}$ en frascos de ámbar depositados en un baño termostatado para el control de la temperatura de operación $\left(42^{\circ} \mathrm{C}\right)$. El pH se ajusta entre 2.0 y 2.5 , con una solución $0.5 \mathrm{M}$ de $\mathrm{H}_{2} \mathrm{SO}_{4}$, y se trabajó a presión atmosférica. Se agrega simultáneamente a cada muestra $5 \mathrm{~mL}$ de peroxido de hidrogeno al $30 \%$ grado analítico en los diferentes frascos ámbar que contienen la solución de fenol. Muestras de $0.5 \mathrm{~mL}$ de solución fueron tomadas en el transcurso de la oxidación hasta completar un tiempo de reacción de 5 horas.

Diversos estudios (Lucking, 1998; Wang, 2005; Guélou, 2003; Quintanilla, 2005; Sánchez., 2001; Carreño y Rovira 2003; García., 2005) han demostrado que las soluciones de peróxido de hidrógeno y sales ferrosas son capaces de oxidar la materia orgánica y esto debido a la formación de radicales HO. generados por la reacción (1):

$\mathrm{Fe}^{2+}+\mathrm{H}_{2} \mathrm{O}_{2} \rightarrow \mathrm{Fe}^{3+}+\mathrm{HO}^{-}+\mathrm{HO}$

$\mathrm{K}=76 \mathrm{l} / \mathrm{mol} . \mathrm{s}$

Los radicales $\mathrm{HO}$ pueden reaccionar luego por dos vías: la oxidación de $\mathrm{Fe}(\mathrm{II})$ de acuerdo a la reacción (2) (reacción improductiva):

$\mathrm{Fe}^{2+}+\mathrm{HO} \rightarrow \mathrm{Fe}^{3+}+\mathrm{HO}$

Y a través del ataque a la materia orgánica (3):

$\mathrm{RH}+\mathrm{HO}+\mathrm{H}_{2} \mathrm{O} \rightarrow \mathrm{ROH}+\mathrm{H}_{3} \mathrm{O}^{+}$

$\rightarrow$ Productos oxidados.

A pH adecuados (entre 2 y 4), el proceso es potencialmente útil para degradar contaminantes, ya que es muy efectivo para la generación de HO. La ecuación de la reacción auto catalítica completa de la oxidación del fenol con $\mathrm{H}_{2} \mathrm{O}_{2}$ se observa en la reacción (4):

$\mathrm{C}_{6} \mathrm{H}_{5} \mathrm{OH}+14 \mathrm{H}_{2} \mathrm{O}_{2} \rightarrow 6 \mathrm{CO}_{2}+17 \mathrm{H}_{2} \mathrm{O}$

La concentración de $\mathrm{H}_{2} \mathrm{O}_{2}$ es analizada por titulaciones yodo métricas con $\mathrm{KI}$, empleando $0.10 \mathrm{~N} \mathrm{Na}_{2} \mathrm{~S}_{2} \mathrm{O}_{3}$ como agente reductor. 
La ecuación general para representar la cinética de las reacciones catalizadas por superficies esta dada por la reacción (5):

$$
-\mathrm{r}_{\mathrm{H}_{2} \mathrm{O}_{2}}=-\frac{\mathrm{dC}_{\mathrm{H}_{2} \mathrm{O}_{2}}}{\mathrm{dt}}=\mathrm{kC}_{\mathrm{H}_{2} \mathrm{O}_{2}}
$$

Donde $r$ es la velocidad de reacción y $\mathrm{k}$ es la constante cinética de reacción.

\section{RESULTADOS Y DISCUSIÓN.}

Los resultados de la caracterización textural por adsorción de $\mathrm{N}_{2}$ a $77 \mathrm{~K}$ del material precursor previo a la impregnación de hierro se encuentra en la tabla 2 , donde se observa que los diferentes materiales de partida poseen características texturales similares.
De aquí la validez del material para comparar la competitividad de estos en igualdad de condiciones para el desarrollo de la oxidación catalítica de fenol asistida con peroxido de hidrogeno.

La tabla 3 muestra la cantidad de hierro que se logró impregnar sobre cada una de las series de muestras de carbón activado. Esta se calcula con respecto a la diferencia entre la cantidad nominal de hierro adicionado en la solución precursora con la cantidad residual de hierro en la solución después de un tiempo de contacto con las muestras de carbón activado. Esta cantidad varía de acuerdo al tipo de material precursor utilizado como también al tratamiento de este antes del proceso impregnación con hierro.

Tabla 1: Tipo de material utilizado, la serie de muestra y la cantidad utilizada.

\begin{tabular}{|llc|}
\hline Tipo de muestra & Serie & Cantidad [g] \\
\hline $\begin{array}{l}\text { a. Carbón activado comercial pelatizado } \\
\text { (desmineralizado-sulfonado-impregnado con hierro) }\end{array}$ & CAP_DS/Fe & 3,602 \\
$\begin{array}{l}\text { b. Tela activada comercial "Zorflex A" } \\
\text { (impregnada con hierro) }\end{array}$ & TZ_A/Fe & 0,858 \\
$\begin{array}{l}\text { c. Carbón activado químicamente con KOH } \\
\text { (desmineralizado-sulfonado-impregnado con hierro) }\end{array}$ & CAQ-KDS/Fe & 0,536 \\
\hline
\end{tabular}

El máximo porcentaje de impregnación de hierro se logró en el carbón mineral activado químicamente con $\mathrm{KOH}$ (Manto AGR), comparado con las demás muestras. Se podría interpretar este resultado como el papel que tendría la superficie de cada uno de estos materiales frente a la capacidad de recepción del hierro en su superficie además de la influencia de la textura porosa.

El tratamiento previo de desmineralización y sulfonación realizado sobre los carbones activados de origen mineral favorece el hecho de que se deposite más hierro sobre la matriz carbonosa. Sin embargo, no se puede asegurar directamente la disponibilidad de todo este hierro para generar la catálisis en la oxidación del fenol, porque algunas trazas podrían haberse fijado en la matriz carbonosa mediante fuertes asociaciones.

La figura 1 presenta los cambios de concentración del $\mathrm{H}_{2} \mathrm{O}_{2}$ durante la oxidación catalítica. Esta cinética es un indicativo de la actividad catalítica de las series de muestras de carbones activados, en la reacción de oxidación de fenol, comparadas con la reacción auto-catalítica entre el fenol y el peroxido en los niveles de concentración de operación. Se observa una tendencia sistemática hacia la disminución de la concentración del $\mathrm{H}_{2} \mathrm{O}_{2}$ en las primeras 3 horas de tiempo de reacción promovida por el efecto catalítico que generan los sólidos empleados en dicha reacción. Niveles de concentraciones bajas de $\mathrm{H}_{2} \mathrm{O}_{2}$ son alcanzados por las muestras en un tiempo de reacción de 5 horas.

Las muestras que alcanzaron el más bajo nivel de concentración de $\mathrm{H}_{2} \mathrm{O}_{2}\left(2.00 * 10^{-3} \mathrm{M}\right)$ fueron la tela activada impregnada y el carbón activado comercial peletizado impregnados con hierro, donde la modificación de este último sólido, favoreció tanto el deposito de hierro disponible para la reacción en la superficie, como también al desempeño de la superficie especifica en el desarrollo de la catálisis. 
Tabla 2: Caracterización de la estructura porosa del material precursor

\begin{tabular}{|c|c|c|c|c|c|}
\hline $\begin{array}{l}\text { SOPORTE } \\
\text { CATALITICO }\end{array}$ & $\begin{array}{c}\text { Área } \\
\text { BET } \\
\left(\mathrm{m}^{2} / \mathrm{g}\right)\end{array}$ & $\begin{array}{c}\text { Volumen } \\
\text { Microporos } \\
\text { DR } \\
\left(\mathrm{cm}^{3} / \mathrm{g}\right) \\
\end{array}$ & $\begin{array}{c}\text { Volumen } \\
\text { Total } \\
\text { P/Po=0.94 } \\
\left(\mathrm{cm}^{3} / \mathrm{g}\right)\end{array}$ & $\begin{array}{l}\text { Diámetro } \\
\text { promedio } \\
\text { poro }(\mathrm{nm})\end{array}$ & $\begin{array}{c}\% \\
\text { Microporos }\end{array}$ \\
\hline TZ_A (Zorflex A) & 695 & 0,345 & 0,392 & 2,252 & 88 \\
\hline CAP (comercial) & 786 & 0.413 & 0.493 & 2.505 & 84 \\
\hline CAQ (Manto AGR) & 799 & 0,414 & 0,456 & 2,285 & 90 \\
\hline
\end{tabular}

Tabla 3: Cantidad de hierro impregnado.

\begin{tabular}{|c|c|c|c|c|}
\hline SERIE & $\begin{array}{l}\text { Diferencia de Hierro entre la } \\
\text { solución patrón y la restante } \\
{[\mathrm{g}]}\end{array}$ & $\begin{array}{c}\text { Cantidad } \\
\text { Carbón activado } \\
{[\mathrm{g}]}\end{array}$ & $\mathrm{gFe} / \mathrm{g}$ CA & $\begin{array}{c}\% \\
\text { Impregnado }\end{array}$ \\
\hline CAP_DS/Fe & 0,564 & 3,601 & 0,157 & 13 \\
\hline $\mathrm{TZ} \_\overline{\mathrm{A}} / \mathrm{Fe}$ & 1,189 & 0,858 & 1,386 & 58 \\
\hline CAQ-KDS/Fe & 1.495 & 0.536 & 2,786 & 74 \\
\hline
\end{tabular}

Todos los carbones activados incluyendo la tela activada promueven la actividad catalítica ambiente iónico, a la superficie química modificada en la sulfonación, como también al hierro que se encuentra entre los sitios interlaminares haciendo parte de la estructura original de los sólidos porosos de origen mineral.

De acuerdo a los resultados obtenidos es probable la factibilidad del uso y el buen desempeño de manera competitiva de la tela activada comercial impregnada con hierro

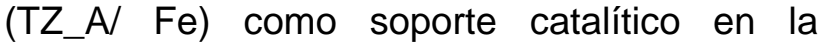
descomposición de fenol frente a otros sólidos porosos convencionales.

Sin embargo, la evaluación de la reutilización de la tela (TZ_A/Fe-R) en la oxidación manifiesta una disminución en la actividad catalítica reflejada en el nivel de concentración de $\mathrm{H}_{2} \mathrm{O}_{2}$ alcanzado.

El tiempo en la disminución de concentración del peroxido de hidrogeno en la reacción auto catalítica con el fenol oscila entre 1 a 2 horas, tal como se muestra en la figura 1; más adelante el cambio alcanzando de los niveles de concentración es prácticamente constante, obteniéndose mediante este ensayo una disminución de concentración de $\mathrm{H}_{2} \mathrm{O}_{2}$ comprendida en el rango alcanzado con respecto a los demás ensayos.

En la tabla 4 se reportan los ordenes de reacción y los coeficientes cinéticos correspondientes para cada ensayo; de aquí que se observa que la mayor parte de los datos de las reacciones catalizadas pueden

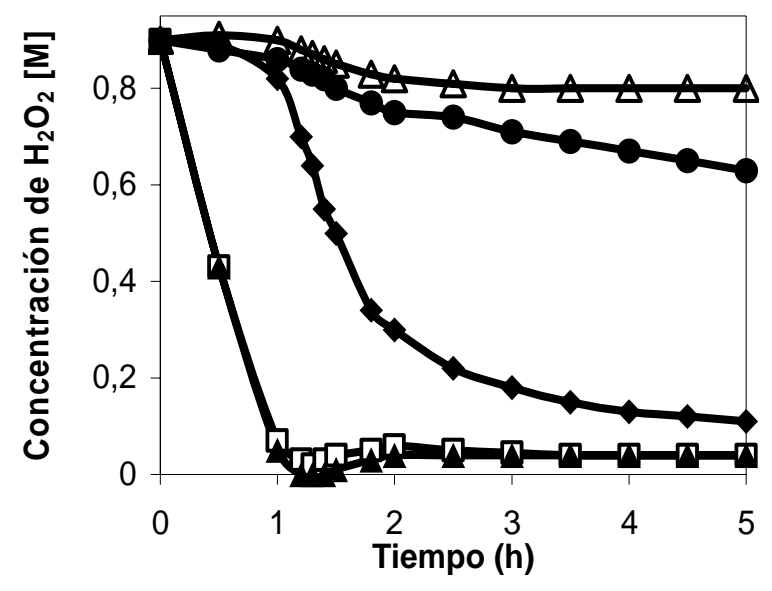

Fig1: Evolución de la concentración del $\mathrm{H}_{2} \mathrm{O}_{2}$ durante la oxidación catalítica. ( $\square-\mathrm{CAP}$ $\mathrm{DS} / \mathrm{Fe} \leadsto-\mathrm{TZ}$ A/Fe $\triangle$-Fenol- $\mathrm{H}_{2} \mathrm{O}_{2} \multimap$ CAQ-KDS/Fe $\rightarrow-T Z$ A/Fe-R ).

ajustarse en función del tiempo de contacto mediante expresiones de velocidad de primero, segundo y tercer orden, relativamente simples de acuerdo al hecho que se haya limitado a la elección de una expresión de velocidad entre las múltiples expresiones más complicadas deducidas de los mecanismos teóricos. Partiendo de las determinaciones de adsorción, se sabe que una elevación en la presión origina un incremento en la cantidad de sustancias adsorbidas; por consiguiente, si la adsorción pudiera ser la etapa que controla la velocidad, un aumento en la concentración del reactante pudo originar un aumento en la velocidad de reacción. Ahora si el factor determinante fuese la reacción química se podría pensar en la posibilidad de aumentar la concentración de fenol o disminuir la concentración de $\mathrm{H}_{2} \mathrm{O}_{2}$ 
de acuerdo a lo planteado por el mecanismo de doble centro para la reacción $A+B \rightarrow$ productos, para favorecer aún más la velocidad de reacción (5).

Los resultados de la catálisis asistida por la tela activada en la degradación del fenol brinda la posibilidad de optimizar el proceso de oxidación a través de la modificación de la cantidad de hierro impregnado, la variación en las condiciones de operación tales como la temperatura y la presión, así como también la variación de la concentración de peroxido a utilizar y de esta manera establecer el mecanismo que mejor represente la velocidad de reacción.

Esto podría llevar a una disminución en el uso de la cantidad de reactivos, lo que generaría una factibilidad en aplicaciones a escala industrial.

Tabla 4: Orden de reacción con respecto al $\mathrm{H}_{2} \mathrm{O}_{2}$ en los ensayos.

\begin{tabular}{|l|c|c|c|}
\hline \multirow{2}{*}{ SERIE } & \multicolumn{2}{|c|}{ Orden de reacción } & \multirow{2}{*}{ K cinética } \\
\cline { 2 - 3 } & Método Integral & $\mathrm{R}^{2}$ & $0.229 \mathrm{~min}^{-1}$ \\
CAP_DS/Fe & 1 & 0.989 & $0.188 \mathrm{~min}^{-1}$ \\
TZ_A/Fe & 1 & 0.992 & $10.96 \mathrm{~min}^{-1}\left(\mathrm{molL}^{-1}\right)^{-2}$ \\
CAQ-KDS/Fe & 3 & 0.977 & $0.014 \mathrm{~min}^{-1} \mathrm{~mol}^{-1} \mathrm{~L}$ \\
Fenol $+\mathrm{H}_{2} \mathrm{O}_{2}$ & 2 & 0.881 & $0.110 \mathrm{~min}^{-1}$ \\
TZ_A/Fe-R & 1 & 0.999 & 0.110 \\
\hline
\end{tabular}

\section{CONCLUSIONES}

La impregnación de hierro mediante una disolución saturada al $20 \%$ en peso de $\mathrm{FeSO}_{4} .7 \mathrm{H}_{2} \mathrm{O}$ sobre la superficie de la tela de carbón activado afecta positivamente la de la cinética en la evolución del $\mathrm{H}_{2} \mathrm{O}_{2}$. El uso de la tela activada como soporte catalítico en la oxidación de fenol resultó ser positivo y competitivo ante otros materiales porosos empleados bajo las mismas condiciones. Este a su vez brinda la opción de ser empleado para otras aplicaciones catalíticas como soporte.

\section{REFERENCIAS}

Das, D, Gaur, V, Verma, N, Removal of volatile organic compound by activated carbon fiber. Carbon 42 , 2949-2962 (2004).

Esplugas, S., Gimenez, J., Contreras, S., Pascual, E. and Rodriguez, M. Comparison of different advanced oxidation processes for phenol degradation. Water Research 36(4): 1034-1042. (2002)

Fortune, W. B. and Mellon, M. G., Determination of iron with O-phenanthroline. A spectrophotometric study. Ind. Eng. Chem. Anal. Ed.10, 60-64 (1938).

Guélou, E, Barrault, J, Fournier, J, Tatibouët, J. M., Active iron species in the catalytic wet peroxide oxidation of phenol over pillared clays containing iron. Applied Catalysis B: Environmental 44, 1-8 (2003).
Levenspiel, Octave, Ingeniería de las reacciones químicas. $2^{\mathrm{a}}$ Edición, 505-513, Reverté, Barcelona, (1996).

Lucking, F, Koser, H, Jank, M and Ritter, A, Iron powder, graphite and activated carbon as catalysts for the oxidation of 4-chlorophenol with hydrogen peroxide in aqueous solution. Wat. Res. 32(9), 2607-2614 (1998).

Pignatello, J. J., Oliveros, E. and Mackay, A. Advanced Oxidation Processes for Organic Contaminant Destruction Based on the Fenton Reaction and Related Chemistry. Critical Rev. Environ. Sci. Technol. 36: 1-84. (2006)

Quintanilla, A, Casas, J. A, Zazo, J. A, Mohedano, A. F., Rodriguez, J. J, We air oxidation of phenol at mild conditions with a Fe/activated carbon catalyst., Applied Catalysis B: Environ., 62 115-120, (2005).

Rodríguez R, Francisco. Marsh, H.,Sciences of Carbons Materials, Publicaciones Univ. de Alicante. España, 345-367, (1996).

Rodríguez Reinoso, F, Pastor, A. C., Marsh, H, Martínez, M. A., Preparation of activated carbon cloths from viscous rayon. Part II: physical activation processes, Carbon, 38(3), 379-395, (2003).

Wang, A, Qu, J, Ru, J, Liu, H, Ge, J, Mineralization of an azo dye Acid Red 14 by electro-Fenton's reagent using an activated carbon fiber cathode, Dies and pigments. 45, 227-233, (2005). 